\title{
Diversity of Hypoviruses and Other Double-Stranded RNAs in Cryphonectria parasitica in North America
}

\author{
Tobin L. Peever, Yir-Chung Liu, and Michael G. Milgroom
}

Department of Plant Pathology, Cornell University, Ithaca, NY 14853.

Current address of T. L. Peever: University of Florida, CREC, 700 Experiment Station Road, Lake Alfred 33850.

Accepted for publication 23 May 1997.

\begin{abstract}
Peever, T. L., Liu, Y.-C., and Milgroom, M. G. 1997. Diversity of hypoviruses and other double-stranded RNAs in Cryphonectria parasitica in North America. Phytopathology 87:1026-1033.

Double-stranded (ds) RNAs in Cryphonectria parasitica were randomly sampled from nine subpopulations in North America using an antibody-based detection system for dsRNA. dsRNA was detected in $166(28 \%)$ of a total of $595 \mathrm{C}$. parasitica isolates sampled by immunoblotting. Incidence of dsRNA infection within subpopulations ranged from $0 \%$ in samples from New Hampshire and Ontario to $100 \%$ in County Line, MI. Most of the dsRNAs sampled were approximately 9 to $13 \mathrm{~kb}$ in size. dsRNAs from 72 isolates analyzed by probing Northern blots with ${ }^{32} \mathrm{P}$-labeled dsRNAs were in one of three hybridization groups. One hybridization group was widespread throughout eastern North America, being found in New York, New Jersey, Maryland, West Virginia, Kentucky, and Michigan. These dsRNAs hybridized to dsRNA from the previously described C. parasitica isolate SR2 from Maryland and are referred to as SR2-type

One dsRNA sampled from Kentucky hybridized to CHV3-type dsRNAs from Michigan. This dsRNA was probably derived from a fungal isolate that had been intentionally released for biological control at this same site 10 years previously and had become established in Kentucky. The third hybridization group was found only in New Jersey. These dsRNAs were much smaller than all other dsRNAs ( 3 and $5 \mathrm{~kb}$ ) and were found in all 11 isolates that were probed; two of these isolates also had SR2-type dsRNA in mixed infections. None of the North American dsRNAs hybridized to CHV1 from Europe, even though CHV1 has been released in numerous locations in eastern North America for biological control of chestnut blight. Similarly, no dsRNAs hybridized to CHV2-NB58, a hypovirus found previously in New Jersey. Mixed infections of SR2-type and CHV3-type dsRNAs were found in 13 of 15 isolates from Frankfort, MI, while another nearby subpopulation (County Line) was infected with only CHV3-type dsRNAs. The distribution of dsRNA hybridization groups in $C$. parasitica thus presents a mixed picture, since one hybridization group is widespread, whereas two others are primarily restricted to smaller geographic areas.
\end{abstract} dsRNAs. The second hybridization group was found almost exclusively in Michigan. The Michigan dsRNAs cross-hybridized to Cryphonectria hypovirus 3-GH2 (CHV3-GH2) and are referred to as CHV3-type dsRNAs.
Additional keywords: biocontrol, Castanea dentata, Endothia parasitica, fungal virus, hypovirulence.
Biological control of chestnut blight in Europe and North America has been associated with hypovirulent isolates of Cryphonectria parasitica (Murrill) Barr that are cytoplasmically infected with double-stranded (ds) RNA hypoviruses (17-19,28,43). Transmission of hypoviruses between isolates of $C$. parasitica through hyphal anastomoses, converting the recipient isolate to hypovirulence, forms the basis for biological control in this system $(3,43)$. Hypovirulence has become established naturally in populations of C. parasitica in Europe and Michigan, and many chestnut trees in these areas are recovering from blight $(17,18)$. Despite these successes, intentional releases of both European and North American hypoviruses in different $C$. parasitica populations in eastern North America have failed to control chestnut blight (28). Successful implementation of biological control of chestnut blight may be aided by a better understanding of the population biology and genetic variation of $C$. parasitica and its associated dsRNAs. Progress has been made in understanding the population genetic structure, mating system, and hypovirus transmission in natural populations of $C$. parasitica $(4,7,26,27,29-32)$, but less is known about variation in the dsRNAs in $C$. parasitica.

Many dsRNAs have been identified from $C$. parasitica isolates, and several have been recognized as the genomes of different members of the virus family Hypoviridae (19), which are phylogenetically related to plant potyviruses (24). The best-studied mem-

Corresponding author: M. G. Milgroom; E-mail address: mgm5@cornell.edu

Publication no. P-1997-0731-01R

(C) 1997 The American Phytopathological Society ber of the family Hypoviridae is Cryphonectria hypovirus 1-EP713 (CHV1-EP713) $(5,39)$, originally isolated from France. Two additional hypoviruses are CHV2-NB58 from New Jersey $(20,21)$ and CHV3-GH2 from Michigan $(11,34,41,42)$. In addition to these members of the family Hypoviridae, several other dsRNAs have been found in $C$. parasitica in North America. A relatively uncharacterized dsRNA from Maryland isolate SR2 is in the size range of hypovirus genomes (16), although it is currently unknown whether SR2 dsRNA belongs to the same family. A mitochondrionassociated dsRNA in isolate NB631 from New Jersey (35), a multicomponent dsRNA in isolate C18 from West Virginia (15), and dsRNA from isolate RC1 from Michigan (17) all appear to be unrelated to the family Hypoviridae.

A few studies have addressed relationships among dsRNAs from various locations in North America and Europe. dsRNAs from different chestnut populations in Europe appear to represent a single species, CHV1 $(21,25)$. CHV1-type dsRNA does not hybridize to North American dsRNAs $(15,16,21,25,34)$ with the exception of CHV2-NB58, to which it cross-hybridizes weakly (21). Similarly, CHV2-NB58 does not hybridize to any North American dsRNAs outside of central New Jersey, including samples of dsRNAs from Michigan, Maryland, Pennsylvania, Virginia, West Virginia, and Tennessee $(15,16,21)$. A Michigan hypovirus (CHV3GH2) hybridized to five of six other Michigan dsRNAs, but did not hybridize to CHV1 nor to dsRNAs from Tennessee and West Virginia (34).

Only two studies provide evidence for cross-hybridization of dsRNAs outside of local populations: Enebak et al. (16) showed that dsRNA from isolate SR2 from Maryland hybridized to dsRNAs 
from 26 C. parasitica isolates collected in Maryland, Virginia, and West Virginia; and L'Hostis et al. (25) showed cross-hybridization between dsRNA from a $C$. parasitica isolate from Michigan (EP60) and dsRNA from an isolate collected in Virginia (EP700). Previous hybridization studies, therefore, suggest substantial diversity for dsRNA and differentiation of hybridization groups among locations within North America.

In addition to the studies on dsRNA diversity cited above, genetic differentiation of $C$. parasitica populations (31) also suggests that its dsRNAs have the potential to be subdivided. dsRNAs are dependent on $C$. parasitica for migration among subpopulations; therefore, the genetic subdivision of $C$. parasitica should give a first approximation to the degree of genetic differentiation expected among dsRNAs. A moderate level of genetic differentiation has been observed among $C$. parasitica subpopulations in North America, suggesting that the population structure is maintained by a combination of restricted gene flow and random genetic drift (31). Based on genetic differentiation of the fungus population, we predicted that dsRNAs would also be differentiated among subpopulations.

Although previous studies have identified distinct hybridization groups of dsRNAs in $C$. parasitica, there may be more diversity than has previously been described. The majority of dsRNAs that have been studied to date in $C$. parasitica have pronounced effects on the morphology of the fungus in culture $(1,6,10,12)$; identifying dsRNA-infected isolates by abnormal cultural morphology may bias samples of dsRNAs against those that have little or no effect on morphology, e.g., dsRNAs like those in isolate SR2 (16) would be under-represented in samples based on abnormal morphology. Similarly, preferential sampling of $C$. parasitica from chestnut blight cankers with a swollen or superficial appearance $(18,21)$ may introduce an additional source of bias in favor of dsRNAs that reduce virulence of $C$. parasitica. A further limitation in describing dsRNA diversity in $C$. parasitica is that, with one exception (16), hybridization studies have been conducted on small numbers of dsRNAs. To understand the relative abundance of different dsRNAs, random samples are needed from several populations.

The objective of this study was to determine the diversity and distribution of hybridization groups of dsRNAs found in C. parasitica in North America. To reduce the sampling biases mentioned above, we used an antibody-based detection method for dsRNAs (38) to screen large numbers of isolates for the presence of dsRNA, regardless of canker or colony morphology. Because we could screen large numbers of isolates with this method, we were able to obtain random samples and estimate the incidence of dsRNAs in nine C. parasitica subpopulations in North America.

\section{MATERIALS AND METHODS}

C. parasitica isolates. Isolates of $C$. parasitica were randomly sampled from nine geographically separated subpopulations of American chestnut, Castanea dentata (Marsh.) Borkh., affected by chestnut blight in eastern North America (Table 1). Seven samples were obtained for previous studies of the population genetic structure of $C$. parasitica $(26,30-32)$. Isolates were sampled from cankers without consideration of canker morphology. Only one isolate was sampled per canker and from one canker per tree to avoid sampling clonally produced cankers (30).

Immunoblot detection of dsRNAs. $C$. parasitica isolates were screened for the presence of dsRNA using an immunoblot procedure with a monoclonal antibody specific for dsRNAs (38). Isolates of $C$. parasitica were grown for 10 to 14 days on potato dextrose agar (PDA) (Difco Laboratories, Detroit) overlaid with sterile cellophane (2). Mycelium was lyophilized, ground to a fine powder, and $900 \mu \mathrm{l}$ of lysis buffer $(200 \mathrm{mM} \mathrm{NaCl} ; 100 \mathrm{mM}$ Tris, $\mathrm{pH}$ $8 ; 2 \%$ sodium dodecyl sulfate [SDS]; and $2 \% \beta$-mercaptoethanol) was added to approximately $50 \mathrm{mg}$ of ground mycelium in $1.9-\mathrm{ml}$ centrifuge tubes. Samples were vortexed briefly, and $900 \mu \mathrm{l}$ of buffered phenol/chloroform (1:1, vol/vol) was added and vortexed again for $20 \mathrm{~s}$. Tubes were spun at $15,000 \times g$ for $10 \mathrm{~min}$ in a microcentrifuge, and the aqueous phase was transferred to $1.5-\mathrm{ml}$ tubes. Five hundred microliters of isopropanol was added to each tube, and samples were mixed gently and spun at $15,000 \times g$ for $10 \mathrm{~min}$ in a microcentrifuge. Supernatants were discarded, and pellets were washed twice in $70 \%$ ethanol. Pellets were dried for 30 min under vacuum and resuspended in $100 \mu \mathrm{l}$ of $0.5 \mathrm{M} \mathrm{NaCl}$ and 4 $\mathrm{mM} \mathrm{MgCl}{ }_{2}$. Half $(50 \mu \mathrm{l})$ of each total nucleic acid (TNA) extract was loaded onto $0.7 \%$ agarose gels $(0.5 \times$ Tris-borate-EDTA [TBE] buffer) and electrophoresed at $3.2 \mathrm{~V} / \mathrm{cm}$ for $3 \mathrm{~h}$. Gels were routinely loaded with TNA extracts of known dsRNA-positive and dsRNA-negative isolates as controls.

Following electrophoresis, nucleic acids were transferred to nylon membranes (Hybond $\mathrm{N}^{+}$; Amersham Corp., Arlington Heights, IL) in $10 \times \mathrm{SSC}(1 \times \mathrm{SSC}$ is $0.15 \mathrm{M} \mathrm{NaCl}$ plus $0.015 \mathrm{M}$ sodium citrate) by capillary blotting; membranes were rinsed in $2 \times \mathrm{SSC}$ and baked $2 \mathrm{~h}$ at $80^{\circ} \mathrm{C}$. Membranes were blocked in phosphatebuffered saline (PBS) $(100 \mathrm{mM} \mathrm{NaCl}$ and $100 \mathrm{mM}$ phosphate buffer, $\mathrm{pH} 7.5$ ) with $0.1 \%$ (vol/vol) Tween 20 (PBS-Tween) and 5\% (wt/vol) skim milk powder for $1 \mathrm{~h}$ at $20^{\circ} \mathrm{C}$ on a rocking platform. Membranes were rinsed twice for $10 \mathrm{~s}$ in PBS-Tween and placed in a hybridization tube with $2.8 \mu \mathrm{g}$ of $\mathrm{J} 2$ anti-dsRNA monoclonal antibody (Phytotest GmbH \& Co., Hilden, Germany) in $10 \mathrm{ml}$ of $\mathrm{PBS}$ and $2 \%(\mathrm{wt} / \mathrm{vol}$ ) bovine serum albumin (BSA). J2 antibody is specific to the structure of dsRNA, regardless of nucleotide sequence (38). Reactions were carried out for $1 \mathrm{~h}$ at $25^{\circ} \mathrm{C}$ in a hybridization oven (Hybaid Instruments, Holbrook, NY). Membranes were rinsed briefly in PBS-Tween, followed by a single 15-min wash and two 5-min washes in PBS-Tween on a rocking platform at $20^{\circ} \mathrm{C}$. Membranes were then incubated with a $1: 10,000$ dilution of horseradish peroxidase-conjugated anti-mouse Ig (Amersham NA 931; Amersham Corp.) in PBS and 2\% (wt/vol) BSA on a rocking platform at $20^{\circ} \mathrm{C}$ for $45 \mathrm{~min}$. Membranes were washed as above by rinsing briefly in PBS-Tween, followed by a single 15-min wash and four 5-min washes in PBS-Tween on a rocking platform at $20^{\circ} \mathrm{C}$. Detection was by chemiluminescence according to manufacturer's instructions (Amersham RPN 2109; Amersham

TABLE 1. Samples of Cryphonectria parasitica from nine subpopulations in North America

\begin{tabular}{|c|c|c|c|}
\hline Subpopulation $^{\mathrm{a}}$ & Location & Collection date & Collector \\
\hline Kentucky & $\begin{array}{l}\text { Natural Bridge State Park, } \\
\text { Powell County, KY }\end{array}$ & July 1993 & L. Shain \\
\hline Maryland & $\begin{array}{l}\text { Finzel, Allegany County, } \\
\text { MD }\end{array}$ & July 1991 & $\begin{array}{l}\text { A. Webb, } \\
\text { E. Seligmann, } \\
\text { M. Double, and } \\
\text { W. MacDonald }\end{array}$ \\
\hline Michigan A & $\begin{array}{l}\text { County Line, Manistee } \\
\text { County, MI }\end{array}$ & June 1986 & A. Michna \\
\hline Michigan B & $\begin{array}{l}\text { Frankfort, Benzie County, } \\
\text { MI }\end{array}$ & June 1986 & A. Michna \\
\hline New Hampshire & $\begin{array}{l}\text { Concord, Merrimack } \\
\text { County, NH }\end{array}$ & January 1993 & M. Milgroom \\
\hline New Jersey & $\begin{array}{l}\text { Earle Naval Weapons } \\
\text { Storage Facility, Five } \\
\text { Points, Monmouth } \\
\text { County, NJ }\end{array}$ & June 1994 & $\begin{array}{l}\text { B. Hillman and } \\
\text { M. Milgroom }\end{array}$ \\
\hline New York & $\begin{array}{l}\text { Danby, Tompkins County, } \\
\text { NY }\end{array}$ & September 1994 & T. Peever \\
\hline Ontario & $\begin{array}{l}\text { Wardsville, Middlesex } \\
\text { County, Ontario, Canada }\end{array}$ & October 1993 & G. Boland \\
\hline West Virginia & $\begin{array}{l}\text { Monongahela National } \\
\text { Forest, Pocahontas } \\
\text { County, WV }\end{array}$ & September 1994 & M. Milgroom \\
\hline
\end{tabular}

a Samples from all subpopulations of $C$. parasitica except New York and West Virginia were from a previous study (31). The New York sample for this study was collected independently, but from an area adjacent to the "BC" subpopulation in Milgroom and Lipari (30,31). 
Corp.). dsRNA was detected as discrete bands or dark smears on the X-ray film (Fig. 1).

Column purification of dsRNA. dsRNA was purified from a sample of isolates that were dsRNA-positive by immunoblotting; a smaller sample of isolates that were dsRNA-negative by immunoblotting was also examined for the presence of dsRNA to evaluate the accuracy of the immunoblot procedure. Isolates were grown in $250 \mathrm{ml}$ of $2.4 \%$ (wt/vol) potato dextrose broth (Difco Laboratories) for 5 days on a rotary shaker at $25^{\circ} \mathrm{C}$ in the dark; shake cultures were started from mycelium scraped from the surface of cultures grown on PDA in the dark to avoid the production of conidia, which may not be uniformly infected with dsRNA $(16,37)$. Approximately $650 \mathrm{mg}$ of lyophilized mycelium of each isolate was ground to a fine powder in liquid nitrogen for each sample. Column purification of dsRNA was performed as described previously (33). dsRNA was resuspended in $50 \mu \mathrm{l}$ of $0.5 \mathrm{M} \mathrm{NaCl}$ and $4 \mathrm{mM} \mathrm{MgCl}_{2}$, treated with $10 \mu \mathrm{g}$ of RNase-free DNAse (Gibco BRL, Gaithersburg, MD) for $30 \mathrm{~min}$ at $37^{\circ} \mathrm{C}$, and stored at $-20^{\circ} \mathrm{C}$.

The quantity of purified dsRNA was assessed using gel electrophoresis. Five microliters of each dsRNA was loaded onto $0.7 \%$ agarose gels $(0.75 \mathrm{mg} / \mathrm{ml}$ of ethidium bromide) with a known quantity of phage lambda DNA, and the gels were run for $2 \mathrm{~h}$ at $8 \mathrm{~V} / \mathrm{cm}$. Quantities of dsRNA were estimated visually by comparing the intensities of bands with lambda DNA when the gel was exposed to UV light.

Northern hybridizations. Approximately $100 \mathrm{ng}$ of each dsRNA was loaded into $0.7 \%$ agarose gels with $0.75 \mathrm{mg} / \mathrm{ml}$ of ethidium bromide and run $16 \mathrm{~h}$ at $1.4 \mathrm{~V} / \mathrm{cm}$ in $0.5 \times$ TBE. After electrophoresis dsRNAs were transferred by capillary-blotting to nylon membrane (Hybond $\mathrm{N}^{+}$; Amersham Corp.) with $50 \mathrm{mM} \mathrm{NaOH}$ for $6 \mathrm{~h}$. Following transfer, membranes were rinsed briefly in $2 \times \mathrm{SSC}$ and baked $2 \mathrm{~h}$ at $80^{\circ} \mathrm{C}$. Purified dsRNA controls from known hybridization groups included CHV1-EP113, CHV1-EP43, CHV2NB58, CHV3-GH2, and isolate SR2. C. parasitica isolates EP113 and EP43 contain dsRNAs derived from French isolate EP3 $(8,12)$;

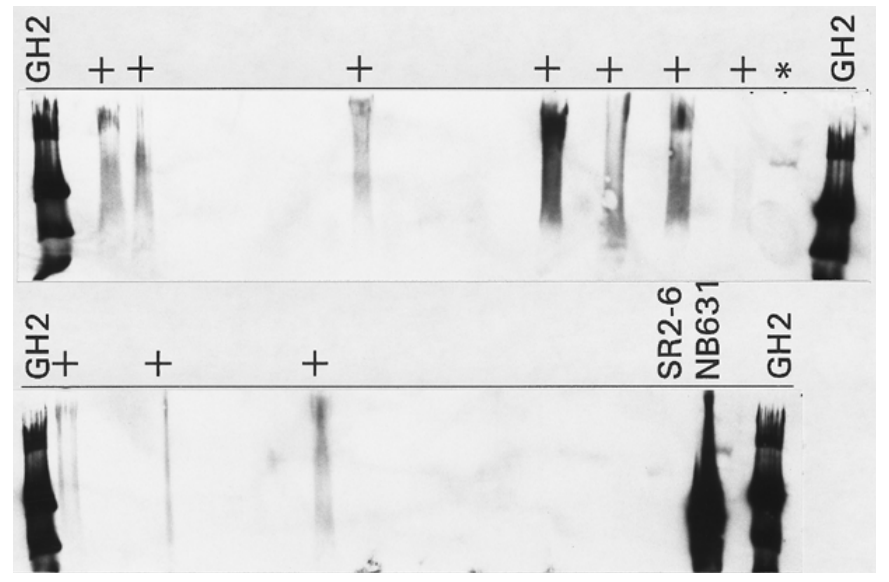

Fig. 1. Immunoblot detection of double-stranded (ds) RNAs with monoclonal antibody $\mathrm{J} 2$ (38) in total nucleic acid extracts from isolates of Cryphonectria parasitica. GH2 and NB631 are $C$. parasitica isolates that were known to be infected with dsRNAs $(34,35)$ and were used as positive controls, SR2-6 is a dsRNA-free single-conidial isolate of SR2 (16) used as a negative control. Isolates labeled + were confirmed as dsRNA-positive by column purification of dsRNA (described in the text). Isolate BC2-43-1 (labeled *) gave a weak signal in the immunoblot, but no dsRNA was detected after column purification (33). Isolates shown were collected in Danby, NY. In the upper panel, isolates in lanes 1 to 26 are $\mathrm{GH} 2, \mathrm{BC} 2-29-2, \mathrm{BC} 2-22-2, \mathrm{BC} 2-39-1, \mathrm{BC} 2-39-2, \mathrm{BC} 2-42-1$, BC2-25-1, ВC2-18-1, BC2-15-1, BC2-23-1, ВC2-29-1, ВC2-1-1, ВC2-11-1, BC2-1-2, ВC2-36-1, ВC2-32-1, BC2-47-2, ВC2-38-1, ВC2-31-1, ВC2-50-1, BC2-27-1, BC2-2-1, BC2-3-1, BC2-43-1, BC2-44-1, and $\mathrm{GH} 2$. In the lower panel, isolates in lanes 1 to 19 are $\mathrm{GH} 2, \mathrm{BC} 2-4-1, \mathrm{BC}-45-1, \mathrm{BC} 2-35-1$, BC2-20-1, BC2-19-1, BC2-24-1, BC2-22-1, BC2-14-1, BC2-21-1, BC2-28-1, BC2-7-1, BC2-10-1, BC2-13-1, BC2-17-1, BC2-47-1, BC2-26-1, BC2-48-1, and BC2-49-1. Isolates in lanes 21 to 24 are SR2-6, NB631, 9B-2-1, and GH2. isolate EP713, which is infected with the well-studied hypovirus CHV1-EP713, is derived from isolate EP155 with the dsRNA from EP113 transmitted into it $(3,22)$. Because EP113 and EP43 are phenotypically similar to EP713 and have dsRNAs from a common source, we considered these viruses to be in the species CHV1 and refer to them as CHV1-EP113 and CHV1-EP43, respectively. Additional dsRNAs used as controls on some gels were purified from isolates NB631 (from B. I. Hillman) (35) and EP102 (from W. L. MacDonald). EP102 was used previously for biological control (9), although it has not been well characterized and no hybridization data are available.

Hybridization groups of dsRNAs were determined using Northern blots of column-purified dsRNA probed with ${ }^{32} \mathrm{P}$-end-labeled dsRNA. Our initial strategy was to randomly choose dsRNAs from several subpopulations to use as probes; later, we also probed with dsRNAs from known hybridization groups. Randomly chosen probes included dsRNA purified from isolates BRU-9-1 (West Virginia), BC2-8-1 (New York), MI-5 (County Line, MI), and NJ116-1 (New Jersey). dsRNA probes from known hybridization groups were purified from CHV1-EP113 or CHV1-EP43 (France), CHV2-NB58 (New Jersey), and from isolate NB631 (New Jersey).

Hybridizations were done essentially as described previously $(23,34)$. dsRNA for use as probes in Northern hybridizations was prepared by an additional column purification as described above. Approximately $1 \mu \mathrm{g}$ of probe dsRNA was hydrolyzed in $100 \%$ formamide at $100^{\circ} \mathrm{C}$ as described previously $(23,34)$ and brought to a final volume of $20 \mu \mathrm{l}$ in sterile $\mathrm{dH}_{2} \mathrm{O}$. Probe dsRNA was endlabeled for $1 \mathrm{~h}$ at $37^{\circ} \mathrm{C}$ with $50 \mu \mathrm{Ci} \gamma^{32} \mathrm{P}$ [ATP] (Dupont, Wilmington, DE), 30 units of T4 polynucleotide kinase, $5 \mu$ of $10 \times$ reaction buffer (United States Biochemical Corp., Cleveland), and $19 \mu \mathrm{l}$ of sterile $\mathrm{dH}_{2} \mathrm{O}$ (total reaction volume of $50 \mu \mathrm{l}$ ). Following labeling, probe dsRNA was purified through Sephadex G50 columns (Sigma Chemical Co., St. Louis) to separate unincorporated label. Membranes were prehybridized for $3 \mathrm{~h}$ at $42^{\circ} \mathrm{C}$ in $50 \%$ formamide; $5 \times \mathrm{SSPE}\left(1 \times \mathrm{SSPE}\right.$ is $0.18 \mathrm{M} \mathrm{NaCl}, 10 \mathrm{mM} \mathrm{NaPO}_{4}$, and $1 \mathrm{mM}$ EDTA [pH 7.7]); $5 \times$ Denhardt's solution; $100 \mu \mathrm{g} / \mathrm{ml}$ of sonicated, denatured salmon sperm DNA; and 0.5\% SDS. Labeled

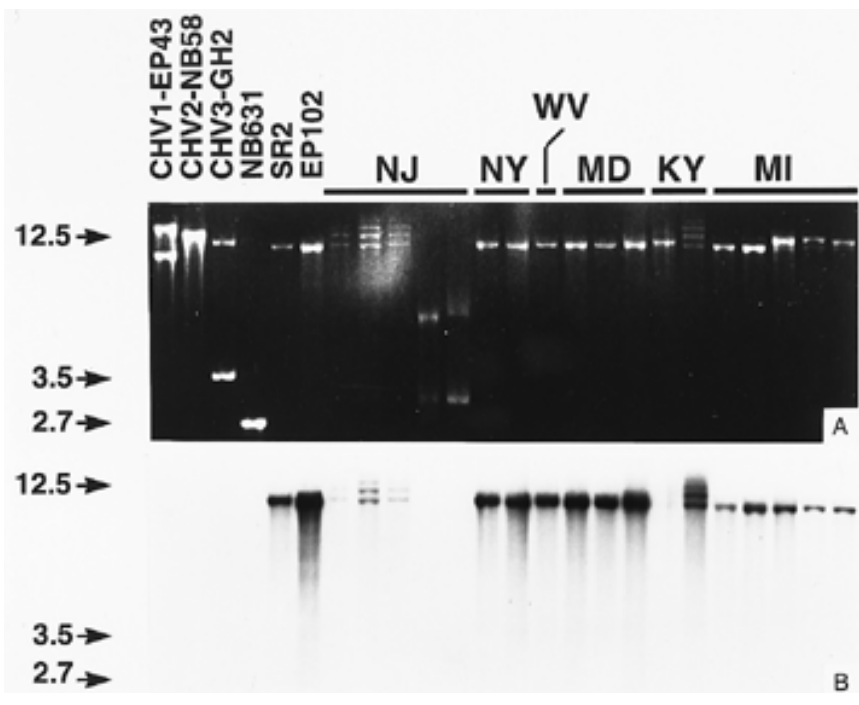

Fig. 2. Hybridization of double-stranded (ds) RNAs from North American to a randomly selected SR2-type dsRNA probe. A, Ethidium bromide-stained agarose gel with dsRNAs of known hybridization groups (lanes 1 to 6 on the left) and dsRNAs sampled from New Jersey (NJ), New York (BC2), West Virginia (BRU), Maryland (MD), Kentucky (KY), and Frankfort, MI (MI) (lanes 7 to 24). B, Northern blot of dsRNAs shown in A probed with ${ }^{32} \mathrm{P}-$ labeled dsRNA from Cryphonectria parasitica isolate BRU-9-1 from West Virginia (SR2-type). Size markers at left indicate positions of dsRNA segments of known size from CHV2-NB58 (12.5 kb [20]) and NB631 dsRNA (2.7 kb [35]), or estimated size from CHV3-GH2 (3.5 kb [42]). Isolates in lanes 7 to 24 are NJ129-2, NJ119-3, NJ120-3, NJ-96-2, NJ116-1, BC2-3-1, BC2-16-1, BRU-9-1, MD1, MD64, MD65, KY11-C-1, KY14, MI18, MI29, MI30, MI31, and MI36. 
probe was added to the prehybridization solution, and hybridizations were carried out overnight at $42^{\circ} \mathrm{C}$. Following hybridization, membranes were washed twice for $5 \mathrm{~min}$ in $2 \times \mathrm{SSC}$ and $0.1 \%$ SDS at $20^{\circ} \mathrm{C}$, followed by two washes for $15 \mathrm{~min}$ in $0.1 \times \mathrm{SSC}$ and $0.1 \% \mathrm{SDS}$ at $50^{\circ} \mathrm{C}$ in a shaking water bath and exposed to $\mathrm{X}$-ray film (Dupont) for 1 to 7 days at $-80^{\circ} \mathrm{C}$ with an intensifying screen. Some membranes were stripped for one additional hybridization by pouring $0.5 \% \operatorname{SDS}\left(100^{\circ} \mathrm{C}\right)$ onto the membrane and letting them cool to room temperature.

\section{RESULTS}

Seven of nine North American subpopulations of $C$. parasitica had detectable dsRNAs by immunoblotting. Isolates sampled from New Hampshire $(n=20)$ and Ontario $(n=33)$ did not contain any detectable dsRNA. Incidence of dsRNA infection in populations with dsRNAs ranged from $6 \%$ in Maryland $(n=69)$ to $100 \%$ in County Line, MI $(n=13)$ (Table 2). The overall incidence of dsRNA infection estimated by immunoblotting was $28 \%$ among 595 isolates (Table 2).

The presence of dsRNAs was confirmed by column purification for a sample of isolates positive for immunoblotting; these purified dsRNA were used for hybridization studies. Column purification failed to yield detectable dsRNA in approximately $15 \%$ of the immunoblot-positive isolates tested (false positives). The false-negative error rate for the immunoblot procedure was estimated in samples from New York and Frankfort, MI. Among 16 New York isolates that were dsRNA-negative by immunoblotting, one (6\%) contained dsRNA detectable by column purification. Five Frankfort isolates tested that were dsRNA-negative by immunoblotting were tested for dsRNA by column purification; all five were dsRNA-positive. Occasionally, this procedure failed to detect dsRNA in isolates that were known to be dsRNA-infected, e.g., isolate 9B-2-1 (15) (Fig. 1).

Most dsRNAs sampled in North America, except in New Jersey, appeared, in our standard electrophoretic conditions, to be single dsRNA segments approximately 9 to $13 \mathrm{~kb}$ in size (Figs. 2 and 3). dsRNAs were purified from 15 New Jersey isolates; all of these isolates had smaller dsRNA segments, approximately 5 and $3 \mathrm{~kb}$ in size, visible in agarose gels (Figs. 2 to 4). Three isolates from New Jersey and one from Kentucky had multiple dsRNA segments in the 9- to 13-kb size range (Fig. 2). No dsRNAs had multiple segments resembling dsRNA from isolate C18 (15). Although most dsRNAs appeared to have single segments in our standard conditions for electrophoresis (Figs. 2 to 4), multiple segments could be seen for some dsRNAs when gels were run for longer times (Fig. 5) (discussed below).

Northern hybridizations. Almost all dsRNAs sampled throughout eastern North America, including dsRNAs from Frankfort, MI, hybridized to a randomly selected dsRNA from West Virginia (isolate BRU-9-1) (Fig. 2B, Table 3); a randomly selected New York dsRNA probe (isolate BC2-8-1) gave identical results to the West

TABLE 2. Proportions of double-stranded (ds) RNA-positive isolates of Cryphonectria parasitica sampled from nine subpopulations in North America as determined by immunoblot detection (38)

\begin{tabular}{lccc}
\hline Subpopulation & $n$ & dsRNA + & Proportion $+^{\mathrm{a}}$ \\
\hline Kentucky & 18 & 3 & 0.17 \\
Maryland & 69 & 4 & 0.06 \\
County Line, Michigan & 13 & 13 & 1.00 \\
Frankfort, Michigan & 17 & 10 & 0.59 \\
New Hampshire & 20 & 0 & 0.00 \\
New Jersey & 259 & 100 & 0.39 \\
New York & 88 & 17 & 0.19 \\
Ontario & 33 & 0 & 0.00 \\
West Virginia & 78 & 19 & 0.24 \\
Total & 595 & 166 & 0.28 \\
\hline
\end{tabular}

a Proportions of isolates screened by immunoblotting that were scored as dsRNA-positive.
Virginia probe. Both of these random probes hybridized to dsRNA from isolate SR2, consistent with a previous report showing that SR2 dsRNA hybridized to dsRNAs found commonly in the central Appalachian region (16). We refer to these dsRNAs as being SR2-type, because they cross-hybridize with SR2 dsRNA.

A randomly selected dsRNA probe from County Line, MI (isolate MI-5), hybridized to dsRNAs from both County Line and Frankfort (Fig. 3D), as well as to CHV3-GH2 (Fig. 3B). The County Line probe did not hybridize to any additional dsRNAs except for one dsRNA from Kentucky (Fig. 3D, Table 3). These dsRNAs represent the second hybridization group found in this study, termed the CHV3-type group.

A third hybridization group was represented by relatively small dsRNAs from New Jersey ( 3 and $5 \mathrm{~kb}$ ). These dsRNAs were almost completely degraded by RNase in a low salt buffer $(0.125 \mathrm{M} \mathrm{NaCl})$, but were not affected by RNase in a high salt buffer $(0.375 \mathrm{M} \mathrm{NaCl})$,
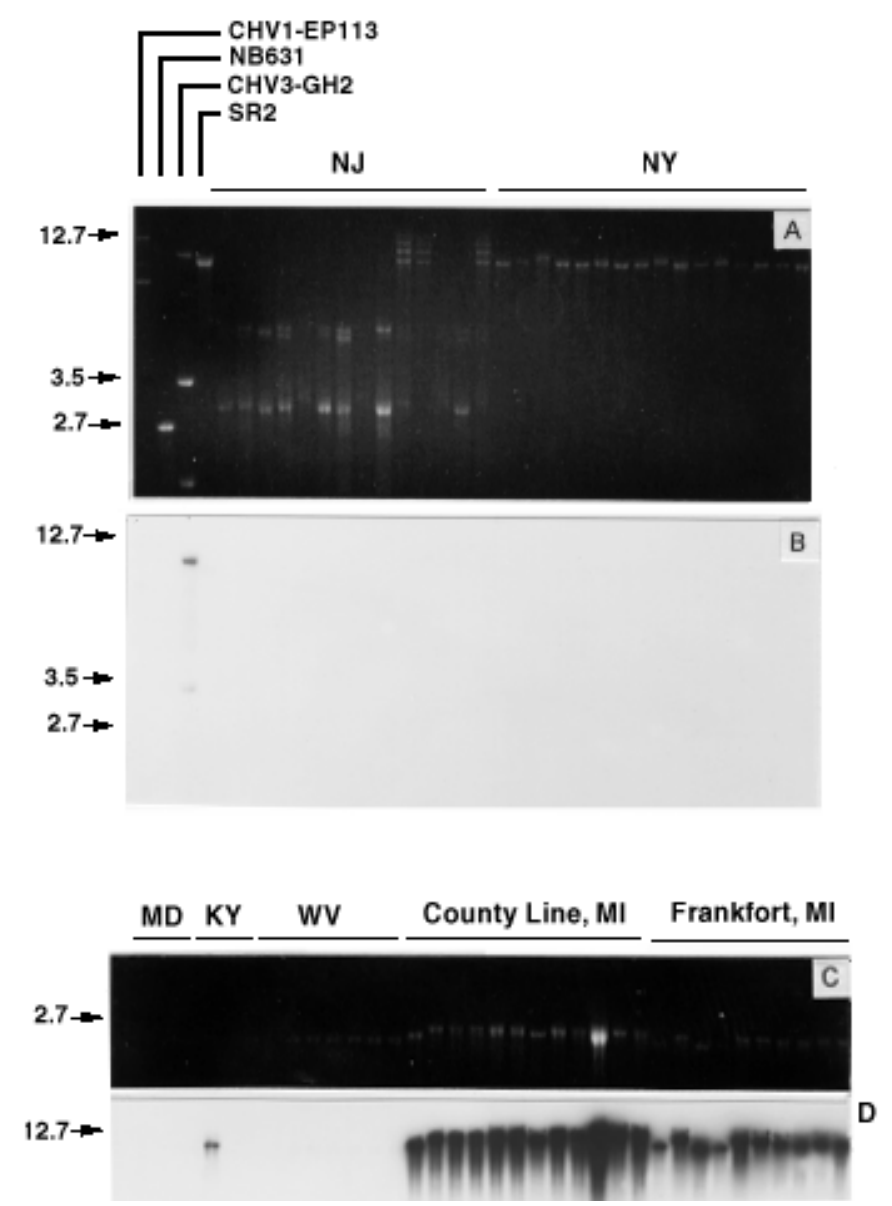

Fig. 3. Hybridization of double-stranded (ds) RNAs from North America to a randomly selected CHV3-type dsRNA probe. Ethidium bromide-stained agarose gel with dsRNAs of known hybridization groups (lanes 1 to 4 ) and dsRNAs sampled from A, New Jersey (NJ), New York (BC2), and Maryland (MD); and C, Maryland (MD), Kentucky (KY), West Virginia (BRU), and County Line (MI1 to MI16) and Frankfort (MI18 to MI36), MI. B and D, Autoradiographs of Northern blots of dsRNAs shown in $\mathbf{A}$ and $\mathbf{C}$, respectively, probed with ${ }^{32} \mathrm{P}$-labeled CHV3-type dsRNA from Cryphonectria parasitica isolate MI-5 from County Line, MI. Size markers at left indicate positions of dsRNA segments of known size from CHV1-EP713 (12.7 kb [39]) and NB631 (2.7 kb [35]) dsRNA, or estimated size from CHV3-GH2 (3.5 kb [42]). Isolates in lanes 5 to 35 in $\mathbf{A}$ and $\mathbf{B}$ are NJ17-3, NJ24-3, NJ32-6, NJ65-3, NJ72-1, NJ85, NJ96-2, NJ112-8, NJ116-1, NJ119-3, NJ120-3, NJ127-2, NJ128-1, NJ129-2, BC2-3-1, BC2-4-1, BC2-6-2, BC2-8-1, BC2-16-1, BC2-20-1, BC2-21-1, BC2-22-2, BC2-23-2, BC2-27-1, BC2-29-1, BC2-30-1, BC2-31-1, BC2-37-1, BC2-39-1, BC2-47-2, and MD1. Isolates in lanes 1 to 36 in $\mathbf{C}$ and $\mathbf{D}$ are MD7, MD64, MD65, KY1, KY11-C-1, KY14, BRU1-1, BRU9-1, BRU14-1, BRU25-1, BRU26-1, BRU36-1, BRU59-1, BRU75-2, MI1, MI4, MI5, MI6, MI7, MI8, MI10, MI11, MI12, MI14, MI15, MI16, MI18, MI19, MI26, MI29, MI30, MI31, MI32, MI34, MI35, and MI36. 
confirming that these segments were dsRNAs. dsRNA probe from isolate NJ116-1 hybridized to 3- and 5-kb segments in all $11 \mathrm{New}$ Jersey isolates that were probed (Fig. 4, Table 3). dsRNAs from other populations were not probed with NJ116-1 dsRNA, because smaller dsRNA segments were not visible on agarose gels for any isolates except those found in New Jersey. These smaller dsRNAs did not hybridize to either SR2-type or CHV3-type dsRNAs (Figs. 2 to 4) or to CHV1-EP43, CHV2-NB58, or NB631 dsRNA probes (Table 3). dsRNA from isolate RC1 from Michigan (34) was also labeled and probed to these small New Jersey dsRNAs and to dsRNA from isolate NB631, but no hybridization was observed (data not shown). No attempts were made to probe with individual segments to determine whether they cross-hybridize.

Mixed dsRNA infections. Two isolates from New Jersey and 13 of 15 isolates from Frankfort, MI, had dsRNAs from two different hybridization groups. dsRNAs from two isolates from New Jersey (NJ129-2 and NJ119-3) hybridized to both SR2-type (Fig. 2) and the 3- and 5-kb dsRNA probes (Fig. 4). Thirteen isolates from Frankfort had dsRNAs that hybridized to both SR2-type (Fig. 2) and CHV3-type probes (Figs. 3D); two isolates from Frankfort hybridized only to SR2-type dsRNA (data not shown). This result was unexpected, because CHV3-type and SR2-type dsRNAs did not cross-hybridize (Figs. 3 and 5). We tested the hypothesis that isolates in Frankfort were infected with dsRNAs from two different hybridization groups by altering electrophoresis conditions to improve separation of dsRNAs $(72 \mathrm{~h}$ at $1.4 \mathrm{~V} / \mathrm{cm})$. The resulting gel was then blotted and probed first with CHV3-type dsRNA (isolate MI-5 from County Line); it was then stripped and reprobed with SR2 dsRNA. Multiple dsRNA segments were visible in most isolates from both County Line and Frankfort (Fig. 5A). The CHV3-type probe hybridized to the larger dsRNA segments in both subpopulations, with the exception of dsRNAs from

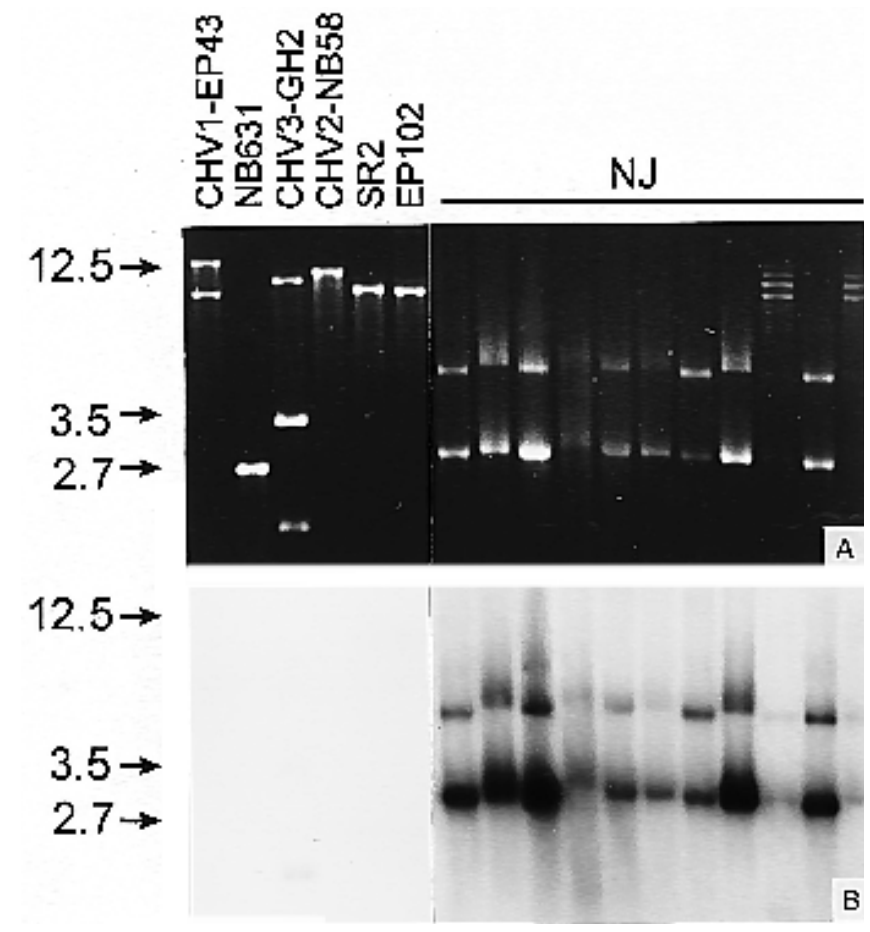

Fig. 4. Hybridization of double-stranded (ds) RNA from New Jersey isolates to labeled dsRNA from isolate NJ116-1. A, Ethidium bromide-stained agarose gel with dsRNAs of known hybridization groups (lanes 1 to 6) and dsRNAs sampled from New Jersey. B, Autoradiograph of Northern blot of dsRNAs shown in A probed with ${ }^{32} \mathrm{P}$-labeled dsRNA from Cryphonectria parasitica isolate NJ116-1 (lane 14). Size marker at left indicates position of dsRNA segments of known size from CHV2-NB58 (12.5 kb [20]) and NB631 (2.7 kb [35]) dsRNA, or estimated size from CHV3-GH2 (3.5 kb [42]). Isolates in lanes 7 to 17 are NJ17-3, NJ24-3, NJ32-6, NJ65-3, NJ72-1, NJ85, NJ96-2, NJ116-1, NJ119-3, NJ128-1, and NJ129-2. three isolates (MI18, MI26, and MI29) that were smaller, approximately the same size as dsRNA from isolate SR2 (Fig. 5). SR2 dsRNA did not hybridize to any County Line dsRNAs, but did hybridize to all of the smaller dsRNA segments in Frankfort, including three segments of the same size that hybridized to CHV3 (Fig. 5C). Therefore, dsRNAs from most isolates in Frankfort represented two hybridization groups, with CHV3-type dsRNAs generally larger than SR2-type dsRNAs. The relative sizes of the largest segment of CHV3-GH2 (approximately $9.6 \mathrm{~kb}$ [11]) and SR2 dsRNA are also visible in gels stained with ethidium bromide (Figs. 2 to 4 ).

Although dsRNAs from every isolate that was probed showed hybridization to one (or two) of three hybridization groups (Table 3), we probed most of the dsRNAs with CHV1-EP113 (or CHV1EP43) and CHV2-NB58, and a smaller sample with dsRNA from NB631, to detect other mixed infections. However, no dsRNAs in our samples hybridized to these additional probes (Table 3). In addition, none of the 100 dsRNA-positive isolates identified by immunoblotting in New Jersey showed the typical colony morphology of isolate NB58 (6), even though CHV2 was previously found in this subpopulation and all CHV2-infected isolates studied had a characteristic morphology $(6,21)$.

\section{DISCUSSION}

Northern hybridizations among dsRNAs from seven subpopulations of $C$. parasitica in North America revealed that all dsRNAs in our samples could be classified into three hybridization groups. One group (SR2-type) was common and widespread throughout
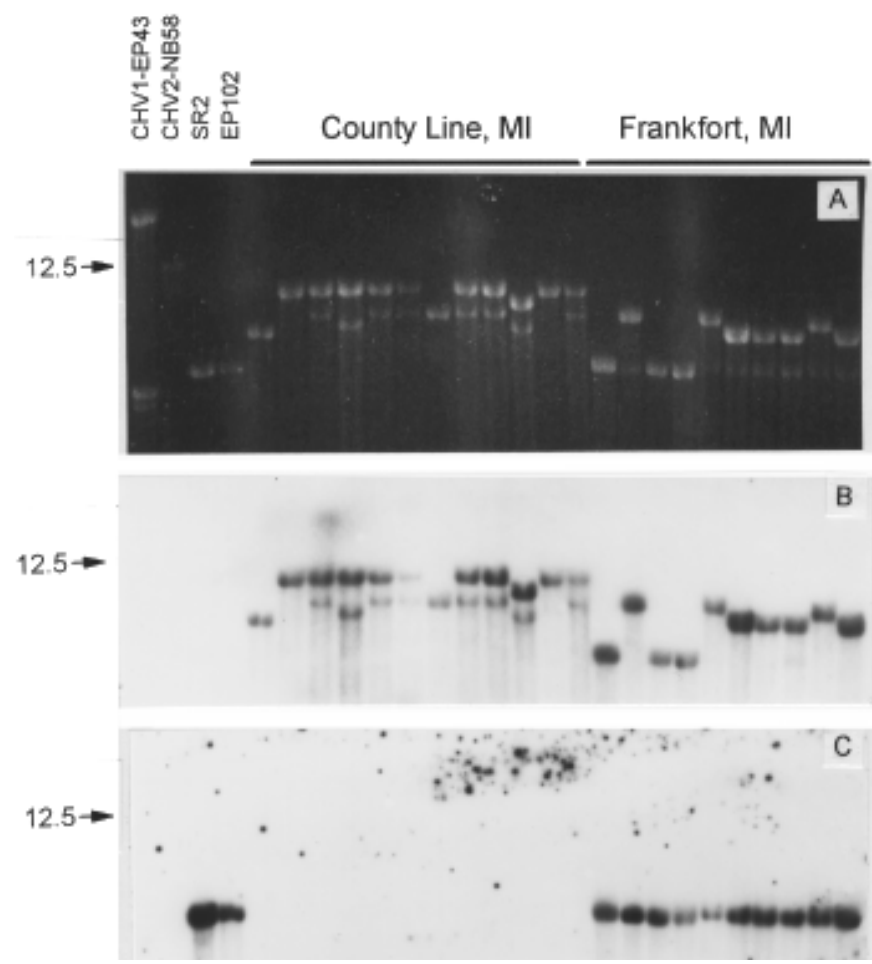

Fig. 5. Hybridization of Michigan double-stranded (ds) RNAs to SR2-type and CHV3-type dsRNA probes. A, Ethidium bromide-stained agarose gel with dsRNAs of known hybridization groups (lanes 1 to 4 ) and dsRNAs sampled from County Line (isolates MI1 to MI16) and Frankfort (isolates MI18 to MI36), MI. Autoradiographs of Northern blots of dsRNAs shown in A probed with ${ }^{32} \mathrm{P}$-labeled B, CHV3-type dsRNA from Cryphonectria parasitica isolate MI-5 from County Line, MI, and C, SR2 dsRNA (16). Size marker at left indicates position of dsRNA segment of known size from CHV2-NB58 (12.5 kb [20]). Electrophoresis for this gel was performed for $72 \mathrm{~h}$ at $1.4 \mathrm{~V} / \mathrm{cm}$ to separate dsRNA segments in different hybridization groups. Isolates in lanes 5 to 26 are MI1, MI4, MI5, MI6, MI7, MI8, MI10, MI11, MI12, MI14, MI15, MI16, MI18, MI19, MI26, MI29, MI30, MI31, MI32, MI34, MI35, and MI36. 
eastern North America; the second was limited primarily to Michigan (CHV3-type); and the third was found only in New Jersey. Results of the current study, indicating that SR2-type dsRNAs are common and widespread in eastern North America, are consistent with previous findings that SR2 dsRNA hybridized to dsRNA from 26 isolates sampled from the central Appalachian region (Virginia, West Virginia, and Maryland) (16). SR2-type dsRNAs have now been identified in at least six different states throughout the native range of the American chestnut, plus Michigan, demonstrating that subpopulations of dsRNAs infecting $C$. parasitica are not necessarily highly differentiated for hybridization groups in North America.

In contrast to SR2-type dsRNAs, the other two hybridization groups were much more limited geographically. The New Jersey hybridization group had not been identified previously and was only observed in one subpopulation. Similarly, CHV3-type dsRNAs were found only in the Michigan subpopulations, plus one isolate from Kentucky. Similar results were obtained by Paul and Fulbright (34) who showed that a Michigan dsRNA (CHV3-GH2) hybridized to five dsRNAs from Michigan, but not to three dsRNAs from Tennessee or West Virginia. We speculate that the one CHV3type dsRNA sampled from Kentucky was derived from intentional releases of CHV3 for biological control in Kentucky (36). Hypovirulent isolates of $C$. parasitica from Michigan were released in Natural Bridges State Park in 1983 (36), the same location from which the $C$. parasitica isolates in this study were sampled 10 years later (Table 1). In West Virginia, CHV3-type hypoviruses have been found in sites where hypovirulent isolates from Michigan had been released 12 to 15 years previously, but were not found immediately outside the treated plots (M. G. Milgroom and W. L. MacDonald, unpublished data). The cross-hybridization found previously (25) between EP60 from Michigan $(13,14)$ and EP700 from Virginia may be another example of CHV3-type dsRNAs found outside of Michigan. EP60 contains multiple dsRNAs (13, 14), some of which hybridize to CHV3-GH2, but not to SR2 dsRNA (Y.-C. Liu and M. G. Milgroom, unpublished data). In addition, dsRNA from isolate D2 from southwestern Pennsylvania, which did not hybridize to SR2 dsRNA in a previous study (16), does hybridize to CHV3-GH2 (Y.-C. Liu and M. G. Milgroom, unpublished data). The lack of CHV1 in our samples demonstrates that European viruses have not become widely established in North America, despite repeated introductions of these hypoviruses (28).

If we had screened $C$. parasitica isolates for morphological effects in culture prior to performing the hybridization studies, we might have obtained a different picture of the diversity of dsRNAs; very few of the isolates we sampled were morphologically abnormal. Using the immunoblot procedure (38), we were able to screen large numbers of $C$. parasitica isolates and sample randomly for dsRNAs, independent of colony morphology. The overwhelming majority of dsRNAs that we sampled in eastern North America belonged to the SR2-type hybridization group, which does not have any noticeable effect on culture phenotype or virulence (16). The immunoblot technique we used saved a significant amount of time and energy compared with screening by dsRNA column purification; however, it was imperfect and sometimes resulted in high error rates. For example, false-negative errors caused us to underestimate the incidence of dsRNA infection in Frankfort, where all five immunoblot-negative isolates tested yielded dsRNAs in column preps. The lower false-negative rate found in New York (one out of 16) indicates that the procedure may be variable and sometimes reasonably accurate. False positives also biased our estimates of dsRNA incidence (approximately 15\%), but were less important in this study because column purification was done for the hybridization studies. Despite these shortcomings, the immunoblot method was useful as a preliminary screen, because it would not have been feasible to screen hundreds of isolates for dsRNAs by column purification. Nonetheless, incidence estimates based on immunoblot results need to interpreted cautiously.
Interestingly, none of the 10 dsRNAs tested in the New Jersey subpopulation hybridized to CHV2-NB58, nor did any of the 100 isolates that were dsRNA-positive by immunoblotting have the characteristic NB58 colony morphology (6). The New Jersey isolates in this study were sampled from the Earle Naval Weapons Storage Facility, adjacent to where NB58- and other CHV2-infected isolates were previously collected $(6,21)$. Furthermore, at least one canker, with abnormal morphology, was sampled within the study area that yielded an isolate infected with CHV2-type dsRNA (B. I. Hillman, personal communication). CHV2-NB58 is reliably detected by both immunoblotting and colony morphology in culture. It is possible that our isolation technique was biased in favor of virus-free single-conidial cultures (16,37); however, we isolated from masses of stomatal tissue (containing large numbers of conidia) or mycelium to avoid single-conidial isolates. The failure of our sampling and screening to identify any isolates with CHV2-type dsRNAs is currently unexplained.

Isolates infected with dsRNAs from two different hybridization groups were common in Frankfort, MI, and New Jersey. Mixed infections have been reported previously in laboratory studies (40) and from two field isolates from Michigan, EP60 $(13,14)$ and GHU4 (34). dsRNAs from isolates EP60 and GHU4 hybridize to CHV3-type dsRNA (34; Y.-C. Liu and M. G. Milgroom, unpublished data), but the identities of additional dsRNAs are not yet known. The result that both CHV3-type and SR2-type dsRNAs hybridized to the same size segment in three Frankfort isolates (Fig. 5) is probably due to comigration of dsRNA segments that are too similar in size to be resolved in these conditions. Furthermore, the titer of CHV3-type dsRNA consistently appeared greater than SR2-type dsRNA, but the intensity of the bands on the gel for these three isolates appeared brighter than the other SR2-type dsRNAs (Fig. 5A), which could be explained by there being two different dsRNA segments comigrating.

The first stage in studying the population genetics of dsRNAs in North America was to describe differentiation at the resolution of hybridization groups. The distribution of dsRNA hybridization groups in C. parasitica presented a somewhat mixed picture. There was a single common hybridization group throughout much of eastern North America; however, two other hybridization groups were locally restricted. Most of the dsRNAs that had been previously identified from different parts of North America were limited in geographic distribution $(15,16,21,34,35)$, suggesting that dsRNA subpopulations were differentiated for hybridization groups in North America. However, the degree of differentiation observed among dsRNA subpopulations in previous studies was only par-

TABLE 3. Hybridization of double-stranded (ds) RNAs from seven subpopulations in North America to ${ }^{32} \mathrm{P}$-labeled dsRNA probes

\begin{tabular}{lcccccc}
\hline & \multicolumn{5}{c}{ Probe dsRNA $^{\mathrm{a}}$} \\
\cline { 2 - 7 } Population & SR2-type $^{\mathrm{b}}$ & CHV3-type & NJ116-1 & CHV1 & CHV2 & NB631 \\
\hline Kentucky & $2 / 3^{\mathrm{d}}$ & $1 / 3$ & $\ldots$ & $0 / 3$ & $0 / 3$ & $0 / 3$ \\
Maryland & $4 / 4$ & $0 / 4$ & $\ldots$ & $0 / 4$ & $0 / 4$ & $0 / 4$ \\
County Line, MI & $0 / 12$ & $12 / 12$ & $\ldots$ & $0 / 12$ & $0 / 12$ & $0 / 3$ \\
Frankfort, MI & $15 / 15$ & $13 / 15$ & $\ldots$ & $0 / 10$ & $0 / 9$ & $0 / 3$ \\
New Jersey & $3 / 14$ & $0 / 14$ & $11 / 11$ & $0 / 10$ & $0 / 10$ & $0 / 6$ \\
New York & $16 / 16$ & $0 / 16$ & $\ldots$ & $0 / 16$ & $0 / 10$ & $0 / 4$ \\
West Virginia & $8 / 8$ & $0 / 8$ & $\ldots$ & $0 / 8$ & $0 / 5$ & $0 / 4$ \\
\hline
\end{tabular}

a Probe dsRNAs were purified from the following Cryphonectria parasitica isolates: BRU-9-1 from West Virginia and BC2-8-1 from New York for SR2-type; MI-5 from Michigan for CHV3-type; and dsRNA from isolate NB631 was previously described (35). For CHV1, we used dsRNA purified from EP713 or EP43; for CHV2, we used CHV2-NB58 (21).

b Identical results were found for both SR2-type probes.

c Isolate NJ116-1, which has only 3- and 5-kb dsRNA segments, was only probed against a subsample of 11 dsRNAs from New Jersey; these smaller dsRNA segments were visible in a total of 15 isolates from New Jersey. Smaller segments of dsRNA $(<9 \mathrm{~kb})$ were not observed except in New Jersey.

${ }^{\mathrm{d}}$ Number of isolates with dsRNA hybridizing/number of dsRNAs probed. 
tially observed in the current study, since SR2-type dsRNAs were even more widespread than described previously (16). Furthermore, little evidence was found for variation in hybridization groups within subpopulations except as mixed infections in New Jersey and Frankfort or in Kentucky, where hypoviruses had been released experimentally.

The high incidence of SR2-type dsRNAs in Frankfort, but not in County Line, MI (approximately $16 \mathrm{~km}$ apart), is an indication that gene flow of dsRNAs is restricted between these two subpopulations. C. parasitica sampled in these same sites were strongly differentiated for restriction fragment length polymorphisms and vegetative compatibility types (26,31). This suggests that both fungus and virus experience little genetic exchange between subpopulations. Random loss of dsRNAs in the SR2-type hybridization group from County Line, without reintroduction from Frankfort or other subpopulations, may have resulted in differentiation between virus populations. Alternatively, SR2-type dsRNAs may only have been introduced into Frankfort, and restricted gene flow may have prevented their introduction into County Line from Frankfort (or elsewhere).

Hybridization studies are only able to provide relatively crude estimates of dsRNA diversity and population structure. However, it is necessary first to show which hybridization groups are present before finer resolution analyses can be performed. Now that it is known that SR2-type dsRNAs are widespread in many different C. parasitica populations in North America, further population genetic studies can be conducted. For example, Chung et al. (6) used a reverse-transcription-polymerase chain reaction (RT-PCR) technique to study the diversity and phylogenetic relationships among a sample of CHV2 from a small area in New Jersey. Unfortunately, the appropriate PCR primers are not yet available for SR2-type dsRNAs. However, the development of primers for SR2-type dsRNA would facilitate further study of their molecular evolution and population structure. Analysis of genetic variation at this level may also shed some light on the origin of this dsRNA, its evolutionary relationship to other dsRNAs infecting $C$. parasitica, and the extent of gene flow in these dsRNAs.

\section{ACKNOWLEDGMENTS}

This research was supported by USDA NRI competitive grant 9537303-1708 and McIntire-Stennis project NYC-153553. We thank S. E. Lipari and Y. Zhou for technical assistance, and B. I. Hillman and C. D. Smart for helpful comments on earlier drafts. We also thank S. L. Anagnostakis, D. W. Fulbright, B. I. Hillman, and W. L. MacDonald for providing us with isolates of Cryphonectria parasitica infected with previously described viruses.

\section{LITERATURE CITED}

1. Anagnostakis, S. L. 1982. Biological control of chestnut blight. Science 215:466-471.

2. Anagnostakis, S. L. 1988. Cryphonectria parasitica: Cause of chestnut blight. Adv. Plant Pathol. 6:123-136.

3. Anagnostakis, S. L., and Day, P. R. 1979. Hypovirulence conversion in Endothia parasitica. Phytopathology 69:1226-1229.

4. Bissegger, M., Rigling, D., and Heiniger, U. 1997. Population structure and disease development of Cryphonectria parasitica in European chestnut forests in the presence of natural hypovirulence. Phytopathology 87:50-59.

5. Choi, G. H., and Nuss, D. L. 1992 Hypovirulence of chestnut blight fungus conferred by an infectious viral cDNA. Science 257:800-803.

6. Chung, P.-H., Bedker, P. J., and Hillman, B. I. 1994. Diversity of Cryphonectria parasitica hypovirulence-associated double-stranded RNAs within a chestnut population in New Jersey. Phytopathology 84:984-990.

7. Cortesi, P., Milgroom, M. G., and Bisiach, M. 1996. Distribution and diversity of vegetative compatibility types in subpopulations of Cryphonectria parasitica in Italy. Mycol. Res. 100:1087-1093.

8. Dodds, J. A. 1980. Association of type 1 viral-like dsRNA with clubshaped particles in hypovirulent strains of Endothia parasitica. Virology 107:1-12.

9. Double, M. L. 1982. The ability of hypovirulent isolates and mixtures of hypovirulent isolates to control artificially established virulent cankers. Pages 145-152 in: Proc. U.S. Dep. Agric. Forest Serv. Am. Chestnut Cooperators Meeting. H. C. Smith and W. L. MacDonald, eds. West Virginia University Books, Morgantown.

10. Dunn, M. M., and Boland, G. J. 1993. Hypovirulent isolates of Cryphonectria parasitica in southern Ontario. Can. J. Plant Pathol. 15:245-252.

11. Durbahn, C. M. 1992. Molecular characterization of dsRNA associated hypovirulence in Michigan isolates of Cryphonectria parasitica. Ph.D. dissertation. Michigan State University, East Lansing.

12. Elliston, J. E. 1985. Characteristics of dsRNA-free and dsRNA-containing strains of Endothia parasitica in relation to hypovirulence. Phytopathology 75:151-158.

13. Elliston, J. E. 1985. Preliminary evidence for two debilitating cytoplasmic agents in a strain of Endothia parasitica from western Michigan. Phytopathology 75:170-173.

14. Elliston, J. E. 1985. Further evidence for two cytoplasmic hypovirulence agents in a strain of Endothia parasitica from western Michigan. Phytopathology 75:1405-1413.

15. Enebak, S. A., Hillman, B. I., and MacDonald, W. L. 1994. A hypovirulent isolate of Cryphonectria parasitica with multiple, genetically unique dsRNA segments. Mol. Plant-Microbe Interact. 7:590-595.

16. Enebak, S. A., MacDonald, W. L., and Hillman, B. I. 1994. Effect of dsRNA associated with isolates of Cryphonectria parasitica from the central Appalachians and their relatedness to other dsRNAs from North America and Europe. Phytopathology 84:528-534.

17. Fulbright, D. W., Weidlich, W. H., Haufler, K. Z., Thomas, C. S., and Paul, C. P. 1983. Chestnut blight and recovering American chestnut trees in Michigan. Can. J. Bot. 61:3164-3171.

18. Heiniger, U., and Rigling, D. 1994. Biological control of chestnut blight in Europe. Annu. Rev. Phytopathol. 32:581-599.

19. Hillman, B. I., Fulbright, D. W., Nuss, D. L., and van Alfen, N. K. 1995. Hypoviridae. Pages 261-264 in: Sixth Report of the International Committee for the Taxonomy of Viruses. F. A. Murphy, C. M. Fauquet, D. H. L. Bishop, S. A. Ghabrial, A. W. Jarvis, G. P. Martelli, M. P. Mayo, and M. D. Summers, eds. Springer Verlag, New York.

20. Hillman, B. I., Halpern, B. T., and Brown, M. P. 1994. A viral dsRNA element of the chestnut blight fungus with a distinct genetic organization. Virology 201:241-250.

21. Hillman, B. I., Tian, Y., Bedker, P. J., and Brown, M. P. 1992. A North American hypovirulent isolate of the chestnut blight fungus with European isolate-related dsRNA. J. Gen. Virol. 73:681-686.

22. Hiremath, S., L'Hostis, B., Ghabrial, S. A., and Rhoads, R. E. 1986. Terminal structure of hypovirulence-associated dsRNAs in the chestnut blight fungus Endothia parasitica. Nucleic Acids Res. 14:9877-9896.

23. Jordan, R. L., and Dodds, J. A. 1983. Hybridization of 5'-end-labelled RNA to plant viral RNA in agarose and acrylamide. Plant Mol. Biol. Rep. 1:31-37.

24. Koonin, E. V., Choi, G. H., Nuss, D. L., Shapira, R., and Carrington, J. C. 1991. Evidence for common ancestry of a chestnut blight hypovirulence-associated double-stranded RNA and a group of positive-strand RNA plant viruses. Proc. Natl. Acad. Sci. U.S.A. 88:10647-10651.

25. L'Hostis, B., Hiremath, S. T., Rhoads, R. E., and Ghabrial, S. A. 1985. Lack of sequence homology between double-stranded RNA from European and American hypovirulent strains of Endothia parasitica. J. Gen. Virol. 66:351-355.

26. Liu, Y.-C., Cortesi, P., Double, M. L., MacDonald, W. L., and Milgroom, M. G. 1996. Diversity and multilocus genetic structure in populations of Cryphonectria parasitica. Phytopathology 86:1344-1351.

27. Liu, Y.-C., and Milgroom, M. G. 1996. Correlation between hypovirus transmission and the number of vegetative incompatibility (vic) genes different among isolates from a natural population of Cryphonectria parasitica. Phytopathology $86: 79-86$.

28. MacDonald, W. L., and Fulbright, D. W. 1991. Biological control of chestnut blight: Use and limitations of transmissible hypovirulence. Plant Dis. 75:656-661.

29. Milgroom, M. G. 1995. Population biology of the chestnut blight fungus, Cryphonectria parasitica. Can. J. Bot. 73(Suppl. 1):S311-S319.

30. Milgroom, M. G., and Lipari, S. E. 1995. Spatial analysis of nuclear and mitochondrial RFLP genotypes in populations of the chestnut blight fungus, Cryphonectria parasitica. Mol. Ecol. 4:633-642.

31. Milgroom, M. G., and Lipari, S. E. 1995. Population differentiation in the chestnut blight fungus, Cryphonectria parasitica, in eastern North America. Phytopathology 85:155-160.

32. Milgroom, M. G., Wang, K., Zhou, Y., Lipari, S. E., and Kaneko, S. 1996. Intercontinental population structure of the chestnut blight fungus, Cryphonectria parasitica. Mycologia 88:179-190.

33. Morris, T. J., and Dodds, J. A. 1979. Isolation and analysis of doublestranded RNA from virus-infected plant and fungal tissue. Phytopathology 69:854-858

34. Paul, C. P., and Fulbright, D. W. 1988. Double-stranded RNA molecules 
from Michigan hypovirulent isolates of Endothia parasitica vary in size and sequence homology. Phytopathology 78:751-755.

35. Polashock, J. J., and Hillman, B. I. 1994. A small mitochondrial doublestranded (ds) RNA element associated with a hypovirulent strain of the chestnut blight fungus and ancestrally related to yeast cytoplasmic $\mathrm{T}$ and W dsRNAs. Proc. Natl. Acad. Sci. U.S.A. 91:8680-8684.

36. Russin, J. S., and Shain, L. 1984. Initiation and development of cankers by virulent and cytoplasmic hypovirulent isolates of the chestnut blight fungus. Can. J. Bot. 62:2660-2664.

37. Russin, J. S., and Shain, L. 1985. Disseminative fitness of Endothia parasitica containing different agents for cytoplasmic hypovirulence. Can. J. Bot. 63:54-57.

38. Schönborn, J., Oberstrab, J., Breyel, E., Tittgen, J., Schumacher, J., and Lukacs, N. 1991. Monoclonal antibodies to double-stranded RNA as probes of RNA structure in crude nucleic acid extracts. Nucleic Acids Res. 19:2993-3000.

39. Shapira, R., Choi, G. H., and Nuss, D. 1991. Virus-like genetic organi- zation and expression strategy for double-stranded RNA genetic elements associated with biological control of chestnut blight. EMBO (Eur. Mol. Biol. Organ.) J. 10:731-739.

40. Smart, C. D., and Fulbright, D. W. 1995. Characterization of a strain of Cryphonectria parasitica doubly infected with hypovirulence-associated dsRNA viruses. Phytopathology 85:491-494.

41. Smart, C. D., Nuss, D. L., and Fulbright, D. W. 1994. Partial sequence analysis of the dsRNA associated with hypovirulence in a Michigan strain of the chestnut blight fungus, Cryphonectria parasitica. Pages 810 in: Proc. Intl. Chestnut Conf. M. L. Double and W. L. MacDonald, eds. West Virginia University Press, Morgantown.

42. Tartaglia, J., Paul, C. P., Fulbright, D. W., and Nuss, D. L. 1986. Structural properties of double-stranded RNAs associated with biological control of chestnut blight fungus. Proc. Natl. Acad. Sci. U.S.A. 83:9109-9113.

43. Van Alfen, N. K., Jaynes, R. A., and Anagnostakis, S. L. 1975. Chestnut blight: Biological control by transmissible hypovirulence in Endothia parasitica. Science 189:890-891. 